\title{
RINGS GENERATED BY THE INNER-AUTOMORPHISMS OF NONABELIAN GROUPS
}

\section{A. JOHN CHANDY}

ABSTRACT. The endomorphisms of an abelian group form a ring. The characterization of groups in which endomorphisms generate a ring is still an open question. It is shown here that the inner-automorphisms of a group generate a ring if and only if the conjugate elements in the group commute.

Groups in which conjugate elements commute have been studied by Levi and Van der Waerden [3] and [4] in connection with the Burnside problem of exponent 3 . We shall call such a group an $L$ group. In this note it is shown that $G$ is an $L$-group, which is nilpotent of class 3 at most, if and only if the inner-automorphisms generate a ring. The endomorphisms of an abelian group form a ring. In general if the group is nonabelian the endomorphisms do not generate a ring. The characterization of groups in which the endomorphisms generate a ring is still an open question.

THEOREM. The inner-automorphisms of a group $G$ generate a ring if and only if the group is an L-group.

Proof. We consider $G$ as a group under addition. Let $A$ be the group of inner-automorphisms of $G$ and $R$ be the near-ring generated by $A$. Suppose $R$ is a ring. Let $a, b, g \in G, g$ fixed, and let $u, v$ be the inner-automorphism of $G$ induced by $a$ and $b$ respectively. We have

$$
\begin{aligned}
(a+g-a)+(b+g-b) & =u g+v g=(u+v) g=(v+u) g \\
& =v g+u g=(b+g-b)+(a+g-a) .
\end{aligned}
$$

Hence the conjugate elements in $G$ commute. Conversely, if $G$ is an $L$-group, then the inner-automorphisms of $G$ commute. But the inner-automorphisms of $G$ generate the additive group $R^{+}$of $R$, hence $R^{+}$must be abelian. So $R$ is a distributively generated nearring with identity whose additive group is abelian and hence $[2$, Theorem 4.4.3] $R$ is a ring.

COROLlaRY. $R$ is a commutative ring if and only if $G$ is nilpotent of class 2.

Presented to the Society, January 25, 1966; received by the editors December 8 , 1970.

AMS 1970 subject classifications. Primary 16A76; Secondary $20 \mathrm{~F} 99$.

Key words and phrases. Nilpotent groups, near-rings, inner-automorphisms.

Copyright $\odot 1971$, American Mathematical Society 
Proof. A group $G$ is nilpotent of class 2 if and only if the factor group with respect to the center (which is isomorphic to the group of inner-automorphisms) is abelian. But since a nilpotent group of class 2 is an $L$-group, the result follows.

\section{REFERENCES}

1. A. Fröhlich, The near-ring generated by the inner automorphisms of a finite simple group, J. London Math. Soc. 33 (1958), 95-107. MR 20 \#67.

2. - Distributively generated near-rings. I. Ideal theory, Proc. London Math. Soc. (3) 8 (1958), 76-94. MR 19, 1156.

3. F. Levi, Groups in which the commutator operation satisfies certain algebraic conditions, J. Indian Math. Soc. 6 (1942), 87-97. MR 4, 133.

4. F. Levi and B. L. Van der Waerden, Über eine besondere Klasse von Gruppen, Abh. Math. Sem. Univ. Hamburg 9 (1933), 154-158.

Southeastern Massachusetts University, North Dartmouth, MassachuSETTS 02747 